\title{
Promotion de la surveillance de la résistance aux antimicrobiens : Résumé du rapport de 2015 du
} CDMTI

\author{
Amaratunga $\mathrm{K}^{1,2}$, Tarasuk $\mathrm{J}^{1}$, Tsegaye $\mathrm{L}^{3}$, Archibald $\mathrm{CP}^{1}$ au nom du Comité directeur sur les \\ maladies transmissibles et infectieuses (CDMTI)* de 2015 du groupe de travail chargé de la \\ surveillance de la résistance aux antimicrobiens ${ }^{4}$
}

\section{Résumé}

Contexte : Les antimicrobiens sont essentiels pour le traitement et le contrôle des maladies infectieuses et, par conséquent, le développement et la propagation de la résistance aux antimicrobiens est un problème de santé mondial. La nécessité d'une surveillance rigoureuse de la résistance aux antimicrobiens est reconnue; toutefois, les lacunes actuelles des programmes de surveillance à l'échelle nationale doivent être comblées afin de permettre la prise de décisions relatives aux programmes et aux politiques mieux fondées sur des données probantes.

Objectif : Décrire la façon dont le groupe de travail chargé de la surveillance de la résistance aux antimicrobiens a classé par ordre de priorité les exigences liées aux données de surveillance nationale en matière d'organismes résistants aux antimicrobiens hautement prioritaires pour la santé humaine au Canada et a formulé des recommandations visant à combler les lacunes actuelles des données.

Méthodologie : Le groupe de travail sur la surveillance de la résistance aux antimicrobiens de 2015 a examiné les exigences en matière de données pour les organismes de priorité de premier ordre déjà identifiés et a déterminé si le système actuel satisfaisait, satisfaisait partiellement ou ne satisfaisait pas à ces exigences. Les renseignements ont été résumés dans des tableaux sommaires et les exigences en matière de données ont été classées par ordre de priorité à l'aide d'un processus de classement afin de formuler des recommandations précises visant à combler les lacunes.

Résultats : Les organismes de priorité de premier ordre pour la surveillance de la résistance aux antimicrobiens sont les suivants: Clostridium difficile, organismes producteurs de $\beta$-lactamase à spectre étendu, organismes résistants aux carbapénèmes (Acinetobacter + espèces Enterobacteriaceae), espèces Enterococcus, Neisseria gonorrhoeae, Streptococcus pyogenes et S. pneumonaea, espèces Salmonella, Staphylococcus aureus, Mycobacterium tuberculosis et espèces Campylobacter. Pour ces organismes, on a déterminé 19 exigences hautement prioritaires en matière de données : 10 de ces exigences étaient satisfaites par les systèmes de surveillance actuels, sept étaient partiellement satisfaites et deux n'étaient pas satisfaites. Pour les deux mesures de données hautement prioritaires dans les milieux communautaires, le groupe de travail a recommandé la réalisation d'une étude de prévalence ponctuelle au sein de la collectivité (c.-à-d. tous les cinq ans) pour effectuer le suivi des taux d'infection à $C$. difficile ainsi que des données d'antibiogrammes à l'échelle des collectivités chaque année pour les données de sensibilité pour les espèces Enterobacteriaceae (E. coli et Klebsiella) causant des infections génito-urinaires. On a déterminé huit exigences modérément prioritaires en matière de données : une exigence était satisfaite par le système de surveillance actuel, cinq étaient partiellement satisfaites et deux n'étaient pas satisfaites. Les exigences insatisfaites en matière de données modérément prioritaires comprenaient la sensibilité des isolats responsables d'infection à C. difficile (maladie diarrhéique) et les taux d'infection par les espèces Enterobacteriaceae causant des infections génito-urinaires dans des milieux communautaires. On a fait remarquer que la faisabilité de l'obtention de ces données modérément prioritaires était faible. Le groupe de travail a mis en évidence les infections du sang comme étant le site d'infection principalement prioritaire pour la surveillance de la résistance aux antimicrobiens dans des milieux de soins de santé, compte tenu des taux élevés de morbidité et de mortalité associés aux infections du sang. L'importance de la collecte de données sur la sensibilité à N. gonorrhoeae dans la collectivité a été soulignée étant donné l'augmentation de la résistance et la collecte seulement partielle de ces données par le système de surveillance actuel. Le groupe de travail a recommandé de procéder à un examen des priorités relatives aux exigences en matière de données de surveillance de la résistance aux antimicrobiens à l'échelle nationale de façon régulière et lorsque de nouveaux problèmes émergent.

Conclusion : Même si les programmes de surveillance nationale actuels saisissent entièrement ou partiellement bon nombre des exigences en matière de données pour les organismes de priorité de premier ordre, il demeure toutefois plusieurs lacunes, en particulier dans les milieux communautaires. Un examen à l'échelle nationale des recommandations du groupe de travail est en cours.

\section{Affiliations}

${ }^{1}$ Centre de la lutte contre les maladies transmissibles et les infections, Agence de la santé publique du Canada, Ottawa (Ontario)

${ }^{2}$ L'Hôpital d'Ottawa et la faculté de médecine de l'Université d'Ottawa, division des maladies infectieuses, Ottawa (Ontario)

${ }^{3}$ Schulich School of Family Medicine and Dentistry, University of Western Ontario, London (Ontario)

${ }^{4}$ Voir la section Remerciements pour obtenir une liste complète des membres du groupe de travail

*Correspondance : cidsc_
secretariat@phac-aspc.gc.ca 
Citation proposée : Amaratunga K, Tarasuk J, Tsegaye L, Archibald CP au nom du Comité directeur sur les maladies transmissibles et infectieuses (CDMTI) de 2015 du groupe de travail chargé de la surveillance de la résistance aux antimicrobiens. Promotion de la surveillance de la résistance aux antimicrobiens : Résumé du rapport de 2015 du CDMTI. Relevé des maladies transmissibles au Canada. 2016;42(11):257-63.

\section{Introduction}

Depuis l'introduction des antibiotiques, on a observé l'émergence de souches résistantes de bactéries. Le développement de la résistance aux antimicrobiens entraîne des obstacles de plus en plus importants et complexes à la prévention, au contrôle et au traitement des maladies infectieuses chez les humains et les animaux.

La surveillance est essentielle pour comprendre l'état actuel et l'évolution de la résistance aux antimicrobiens. Pendant plusieurs années, l'Agence de la santé publique du Canada (ASPC) a travaillé en collaboration avec les provinces et les territoires pour la mise en place d'un certain nombre de programmes de surveillance visant à effectuer le suivi de la résistance aux antimicrobiens et de l'utilisation des antimicrobiens en cours dans les hôpitaux et les milieux communautaires, ainsi que dans les milieux vétérinaires et agricoles (1-4). Un engagement clé du plan d'action fédéral sur la résistance et le recours aux antimicrobiens au Canada est de fusionner les différents systèmes de surveillance dans un point focal commun par l'entremise du Système canadien de surveillance de la résistance aux antimicrobiens (SCSRA), lancé en 2014 (5). Le SCSRA fournit un tableau exhaustif du recours et de la résistance aux antimicrobiens au Canada en présentant des données provenant de neuf systèmes de surveillance et services de référence en laboratoire de I'ASPC et il est maintenant publié chaque année (6). En plus de la lutte contre la résistance aux antimicrobiens chez certaines populations à risque élevé, comme les Autochtones, l'analyse des données de surveillance axée sur le sexe et le genre a été relevée comme point à considérer pour l'avenir.

Le Comité directeur sur les maladies transmissibles et infectieuses (CDMTI) du Conseil du Réseau pancanadien de santé publique (qui représente les partenaires fédéraux, provinciaux et territoriaux) a établi la résistance aux antimicrobiens comme priorité ainsi que la nécessité de robustes systèmes de surveillance pour éclairer l'élaboration de politiques et de programmes efficaces de prévention et de contrôle de la résistance aux antimicrobiens. En 2014, le CDMTI a mis sur pied un groupe de travail composé d'experts afin de formuler des recommandations pour lutter contre les infections courantes acquises en milieu de soins de santé et se pencher sur les questions opérationnelles liées à la surveillance de la résistance aux antimicrobiens. Le groupe de travail a déterminé les éléments clés d'une approche pancanadienne en matière de surveillance de la résistance et du recours aux antimicrobiens pour les aspects liés à la santé humaine et a établi une liste d'organismes prioritaires aux fins de surveillance de la résistance aux antimicrobiens classés selon la priorité d'importance de premier, de deuxième et de troisième ordre (tableau 1).

En 2015, un nouveau groupe de travail du CDMTI composé d'experts, le groupe de travail chargé de la surveillance de la résistance aux antimicrobiens du CDMTI, a été formé pour formuler des conseils et des recommandations sur les exigences en matière de données prioritaires (mesures de données)
Tableau 1 : Organismes prioritaires pris en considération pour la surveillance de la résistance aux antimicrobiens $^{1}$

\begin{tabular}{|c|c|c|}
\hline $\begin{array}{c}\text { Priorité de premier } \\
\text { ordre }\end{array}$ & $\begin{array}{c}\text { Priorité de } \\
\text { deuxième ordre }\end{array}$ & $\begin{array}{l}\text { Priorité de } \\
\text { troisième ordre }\end{array}$ \\
\hline Clostridium difficile & $\begin{array}{l}\text { Espèces } \\
\text { Aspergillus }\end{array}$ & $\begin{array}{l}\text { Espèces } \\
\text { Aeromonas }\end{array}$ \\
\hline $\begin{array}{l}\text { Organismes producteurs de } \\
\text { BLSE }^{2}\end{array}$ & $\begin{array}{l}\text { Espèces } \\
\text { Bacteroides }\end{array}$ & $\begin{array}{l}\text { Chlamydia } \\
\text { pneumoniae }\end{array}$ \\
\hline $\begin{array}{l}\text { Organismes résistants } \\
\text { aux carbapénèmes } \\
\text { (Acinetobacter et espèces } \\
\text { Enterobacteriaceae) })^{3}\end{array}$ & Candida albicans & $\begin{array}{l}\text { Cryptococcus } \\
\text { neoformans }\end{array}$ \\
\hline Espèces Enterococcus & $\begin{array}{l}\text { Chlamydia } \\
\text { trachomatis }\end{array}$ & $\begin{array}{l}\text { Haemophilus } \\
\text { influenza }\end{array}$ \\
\hline Neisseria gonorrhoeae & $\begin{array}{l}\text { Helicobacter } \\
\text { pylori }\end{array}$ & $\begin{array}{l}\text { Mycobactéries } \\
\text { non tuberculeuses } \\
\text { (pulmonaires) }\end{array}$ \\
\hline $\begin{array}{l}\text { Streptococcus pyogenes } \\
\text { Streptococcus pneumoniae }\end{array}$ & $\begin{array}{l}\text { Pseudomonas } \\
\text { aeruginosa }\end{array}$ & \\
\hline Espèces Salmonella & $\begin{array}{l}\text { Streptococcus du } \\
\text { groupe B }\end{array}$ & \\
\hline Staphylococcus aureus & Espèces Shigella & \\
\hline \multicolumn{3}{|l|}{ Mycobacterium tuberculosis } \\
\hline \multicolumn{3}{|l|}{ Espèces Campylobacter } \\
\hline $\begin{array}{l}\text { Tels qu'ils ont été définis et acceptés } \\
\text { résistance aux antimicrobiens du CDM } \\
2 \text { Organismes producteurs de } \beta \text {-lactam } \\
\text { coli), Pseudomonas. Autres organismes } \\
\text { Proteus, Enterobacter } \\
{ }^{3} \text { Organismes résistants aux carbapénè } \\
\text { Pseudomonas, Acinetobacter }\end{array}$ & $\begin{array}{l}\text { groupe de travail ch } \\
\text { décembre } 2014 \text { (rap } \\
\text { LSE) : espèces Enter }\end{array}$ & $\begin{array}{l}\text { de la surveillance de la } \\
\text { on publié) } \\
\text { eriaceae (Klebsiella, E. } \\
\text { ii, Citrobacter, Serratia, } \\
\text { ae (Klebsiella, E. coli), }\end{array}$ \\
\hline
\end{tabular}

nécessaires pour soutenir un rigoureux système de surveillance de la résistance aux antimicrobiens pour chaque organisme de priorité de premier ordre. Le présent article résume les résultats du groupe de travail chargé de la surveillance de la résistance aux antimicrobiens du CDMTI dans le Rapport au CDMTI : Données de surveillance exigées concernant la résistance aux antimicrobiens des organismes prioritaires (7).

\section{Méthodologie}

Les membres du groupe de travail comprenaient des cliniciens spécialisés en maladies infectieuses, des praticiens chargés de la prévention et du contrôle des infections, des microbiologistes médicaux, des praticiens en santé publique et des spécialistes de la résistance aux antimicrobiens canadiens. Le groupe de travail a d'abord passé en revue et résumé les exigences en matière de données de surveillance pour les organismes de priorité de premier ordre. Il a ensuite utilisé un processus de classement afin d'établir les priorités des exigences en matière de données et formulé des recommandations précises visant à combler ces lacunes dans les données de surveillance. 


\section{Phase 1 : Examen des exigences en matière de données de surveillance}

Le groupe de travail chargé de la surveillance de la résistance aux antimicrobiens du CDMTI a passé en revue les exigences suivantes en matière de données de surveillance pour chacun des organismes de priorité de premier ordre :

- $\quad$ Site d'infection (désigne le syndrome ou le type d'échantillon à recueillir [p. ex. infection du sang, infection génito-urinaire, etc.]).

- Source des données (fait référence au système de surveillance qui fournit les données).

- Données sur des variables pertinentes (mesurées par le taux d'infection, le taux de colonisation ou la sensibilité de l'organisme).

- Priorité et pertinence (fait référence à l'importance de cette mesure pour chaque organisme et vise à déterminer s'il s'agit de la mesure la plus appropriée).

- Faisabilité (désigne la possibilité de recueillir les données requises).

- Justification de la mesure (au besoin).

- Antibiotiques à considérer aux fins des tests (au besoin).

- Autres considérations (au besoin).

Pour chaque organisme étudié, le groupe de travail chargé de la surveillance de la résistance aux antimicrobiens du CDMTI a sélectionné le site d'infection jugé comme étant d'importance nationale ou comme étant conforme aux exigences mondiales en matière de surveillance de la résistance aux antimicrobiens de I'Organisation mondiale de la Santé (OMS) (8).

\section{Phase 2 : Élaboration des tableaux sommaires}

Un résumé des discussions a été préparé pour chaque organisme, incluant une description du ou des systèmes de surveillance actuels et les limites de chacun des systèmes. Ce résumé comprenait également un classement subjectif de l'ordre de priorité des exigences en matière de données ainsi que des recommandations fondées sur l'opinion d'experts membres du groupe de travail. Un tableau sommaire a été créé à partir de ces renseignements pour chaque organisme de priorité de premier ordre qui indiquait les éléments suivants :

- Le milieu (soins de santé [p. ex. hôpitaux de soins de courte durée] ou communautaire [c.-à-d. un milieu où les soins de santé primaires sont fournis, y compris les établissements de soins de longue durée]).

- Les mesures de données requises sont les suivantes : - Taux d'infection (incidence et/ou prévalence); - Taux de colonisation (incidence et/ou prévalence);

- Renseignements sur la sensibilité de l'organisme aux antibiotiques.

- $\quad$ Note de priorité de l'exigence en matière de données (élevée, modérée, faible).

- Évaluation déterminant si le système de surveillance actuel répond aux besoins cernés (répond aux besoins, répond partiellement aux besoins, ne répond pas aux besoins ou une brève description si la mesure de données est considérée comme étant une faible priorité).

- Intervention requise pour combler les lacunes décelées, le cas échéant.

- Faisabilité de la mise en œuvre de la nouvelle intervention proposée pour combler la lacune décelée.
Pour les isolats capables d'infecter et de coloniser, le type de sensibilité aux antibiotiques choisi était fondé sur les données de laboratoire accessibles, la pertinence clinique et la déclaration de l'OMS (9). Les données pertinentes en matière de sensibilité ont été fournies sous forme de données sensible/ intermédiaire/résistant (SIR) plutôt que sous forme de données de concentration minimale inhibitrice (CMI).

La tableau 2 présente un exemple de tableau sommaire pour un organisme prioritaire, $C$. difficile; les bandes grises indiquent les milieux de soins de santé et les milieux communautaires sous lesquels se trouvent les types de données requises pour chacun.

Tableau 2 : Exemple de tableau sommaire pour Clostridium difficile (maladie diarrhéique)

\begin{tabular}{|c|c|c|c|}
\hline $\begin{array}{c}\text { Milieu et mesures } \\
\text { de données } \\
\text { requises }\end{array}$ & $\begin{array}{l}\text { Priorité des } \\
\text { mesures de } \\
\text { données }\end{array}$ & $\begin{array}{l}\text { Système de } \\
\text { surveillance } \\
\text { actuel }\end{array}$ & Faisabilité \\
\hline \multicolumn{4}{|l|}{ Milieu de soins de santé } \\
\hline Taux d'infection & Élevée & $\begin{array}{l}\text { Répond aux } \\
\text { besoins }\end{array}$ & Élevée \\
\hline $\begin{array}{l}\text { Sensibilité de } \\
\text { l'isolat responsable } \\
\text { d'infection }\end{array}$ & Modéré & $\begin{array}{l}\text { Répond aux } \\
\text { besoins }\end{array}$ & Élevée \\
\hline $\begin{array}{l}\text { Taux de colonisation } \\
\text { et sensibilité de } \\
\text { l'isolat responsable } \\
\text { de colonisation }\end{array}$ & Faible & $\begin{array}{l}\text { Le système de } \\
\text { surveillance } \\
\text { actuel ne } \\
\text { recueille ou ne } \\
\text { publie aucune } \\
\text { donnée }\end{array}$ & $\begin{array}{l}\text { Non } \\
\text { évaluée }\end{array}$ \\
\hline \multicolumn{4}{|l|}{ Milieu communautaire } \\
\hline Taux d'infection & Élevée & $\begin{array}{l}\text { Ne répond pas } \\
\text { aux besoins }\end{array}$ & Modéré \\
\hline $\begin{array}{l}\text { Sensibilité de } \\
\text { l'isolat responsable } \\
\text { d'infection }\end{array}$ & Modéré & $\begin{array}{c}\text { Ne répond pas } \\
\text { aux besoins }\end{array}$ & Faible \\
\hline $\begin{array}{l}\text { Taux de colonisation } \\
\text { et sensibilité de } \\
\text { l'isolat responsable } \\
\text { de } \\
\text { colonisation }\end{array}$ & Faible & $\begin{array}{l}\text { Le système de } \\
\text { surveillance } \\
\text { actuel ne } \\
\text { recueille ou ne } \\
\text { publie aucune } \\
\text { donnée }\end{array}$ & $\begin{array}{c}\text { Non } \\
\text { évaluée. }\end{array}$ \\
\hline
\end{tabular}

\section{Phase 3 : Détermination des exigences et des recommandations en matière de données sur la priorité}

Un processus en trois étapes a été utilisé afin d'examiner les exigences les plus importantes en matière de données et les prochaines étapes possibles:

Étape 1 : La liste des exigences en matière de données a été stratifiée par priorité (élevée, modérée, faible). Dans ce cas, la priorité désigne une évaluation de l'ensemble de l'importance de l'exigence en matière de données pour la surveillance de la résistance aux antimicrobiens à l'échelle nationale, selon le jugement consensuel des membres du groupe de travail. 
Étape 2 : La liste des exigences en matière de données obtenue à l'étape 1 a été stratifiée davantage selon l'état du système de surveillance actuel. Le groupe de travail a évalué l'état du système de surveillance correspondant en place et a déterminé si le système recueillait ou publiait actuellement des données sur les mesures de données prioritaires établies. Pour chaque exigence de données, le système national de surveillance correspondant actuellement en place a été classé dans une des catégories suivantes : répond aux besoins, répond partiellement aux besoins ou ne répond pas aux besoins.

Étape 3 : La liste des exigences en matière de données issues de l'étape 2 a été stratifiée davantage par une mesure de faisabilité dans les catégories suivantes: faisabilité élevée, modérée, faible ou non évaluée. La faisabilité était déterminée en fonction du degré d'efforts en personnes-temps et des ressources financières nécessaires pour mettre en œuvre l'intervention proposée visant à combler les lacunes en matière de données exigées.

Des recommandations ont été formulées à l'aide de l'examen du processus en trois étapes, d'une discussion et de l'établissement d'un consensus.

\section{Résultats}

\section{Exigences en matière de données hautement prioritaires}

Pour les organismes de priorité de premier ordre, le groupe de travail chargé de la surveillance de la résistance aux antimicrobiens du CDMTI a mis en évidence 19 mesures de données hautement prioritaires nécessaires à la mise en place d'un robuste système de surveillance de la résistance aux antimicrobiens à l'échelle nationale. De ce nombre, 10 étaient satisfaites, sept étaient partiellement satisfaites et deux n'étaient pas satisfaites par le système de surveillance actuel.

\section{Répond aux besoins}

Le groupe de travail a examiné 10 des 19 mesures hautement prioritaires pour lesquelles les systèmes de surveillance existants à l'échelle nationale répondaient aux besoins. Lors de l'évaluation de la faisabilité, huit présentaient une faisabilité élevée de la collecte de données et aucune nouvelle intervention n'était requise (aucune lacune décelée) et deux présentaient une faisabilité modérée de la poursuite de la collecte des données requises, sous réserve de la disponibilité des ressources.

\section{Répond partiellement aux besoins}

Le groupe de travail a ensuite examiné les sept des 19 mesures hautement prioritaires pour lesquelles les systèmes de surveillance existants à l'échelle nationale répondaient partiellement aux besoins (tableau 3). Parmi ces mesures, quatre étaient des infections du sang dans des milieux de soins de santé (sensibilité des isolats responsables d'infection à Enterococcus; les taux d'infection et la sensibilité des isolats responsables d'infection pour les espèces Enterobacteriaceae E. coli et Klebsiella; les taux d'infection de S. aureus; et la sensibilité des isolats responsables d'infection à $S$. aureus); deux se trouvaient dans des milieux communautaires pour d'autres sites d'infection, y compris des infections du sang (sensibilité
Tableau 3 : Mesures de données hautement prioritaires pour lesquelles les systèmes de surveillance actuels répondent partiellement aux besoins

\begin{tabular}{|c|c|c|c|c|}
\hline Organisme & Milieu & $\begin{array}{l}\text { Mesure de } \\
\text { donnée } \\
\text { prioritaire }\end{array}$ & $\begin{array}{l}\text { Système de } \\
\text { surveillance } \\
\text { actuel }\end{array}$ & Faisabilité \\
\hline $\begin{array}{l}\text { Enterococcus : } \\
\text { (Infections du sang - les } \\
\text { infections à ERV sont } \\
\text { considérées comme les } \\
\text { plus importantes) }\end{array}$ & Soins de santé & $\begin{array}{l}\text { Sensibilité } \\
\text { de l'isolat } \\
\text { responsable } \\
\text { d'infection }\end{array}$ & $\begin{array}{l}\text { Répond } \\
\text { partiellement } \\
\text { aux besoins }\end{array}$ & Élevée \\
\hline $\begin{array}{l}\text { Espèces } \\
\text { Enterobacteriaceae : } \\
\text { Escherichiae coli et } \\
\text { Klebsiella (infections du } \\
\text { sang) }\end{array}$ & Soins de santé & $\begin{array}{l}\text { Taux } \\
\text { d'infection } \\
\text { et } \\
\text { sensibilité } \\
\text { de l'isolat } \\
\text { responsable } \\
\text { d'infection }\end{array}$ & $\begin{array}{l}\text { Répond } \\
\text { partiellement } \\
\text { aux besoins }\end{array}$ & Modérée \\
\hline $\begin{array}{l}\text { Staphylococcus aureus } \\
\text { (infections du sang) }\end{array}$ & Soins de santé & $\begin{array}{l}\text { Taux } \\
\text { d'infection }\end{array}$ & $\begin{array}{l}\text { Répond } \\
\text { partiellement } \\
\text { aux besoins }\end{array}$ & Modérée \\
\hline $\begin{array}{l}\text { S. aureus (infections du } \\
\text { sang) } \\
\text { (les infections à SARM } \\
\text { sont considérées comme } \\
\text { les plus importantes) }\end{array}$ & Soins de santé & $\begin{array}{l}\text { Sensibilité } \\
\text { de l'isolat } \\
\text { responsable } \\
\text { d'infection }\end{array}$ & $\begin{array}{l}\text { Répond } \\
\text { partiellement } \\
\text { aux besoins }\end{array}$ & Modérée \\
\hline $\begin{array}{l}\text { S. aureus (autres sites } \\
\text { d'infection, y compris } \\
\text { les infections du sang, et } \\
\text { sites de colonisation) } \\
\text { (les infections à SARM } \\
\text { sont considérées comme } \\
\text { les plus importantes) }\end{array}$ & Communautaire & $\begin{array}{l}\text { Sensibilité } \\
\text { de l'isolat } \\
\text { responsable } \\
\text { d'infection }\end{array}$ & $\begin{array}{l}\text { Répond } \\
\text { partiellement } \\
\text { aux besoins }\end{array}$ & Modérée \\
\hline $\begin{array}{l}\text { Streptococcus } \\
\text { pneumoniae (maladie } \\
\text { invasive) }\end{array}$ & $\begin{array}{l}\text { Soins de } \\
\text { santé et } \\
\text { communautaire }\end{array}$ & $\begin{array}{l}\text { Sensibilité } \\
\text { de l'isolat } \\
\text { responsable } \\
\text { d'infection }\end{array}$ & $\begin{array}{l}\text { Répond } \\
\text { partiellement } \\
\text { aux besoins }\end{array}$ & Modérée \\
\hline Neisseria gonorrhoeae & Communautaire & $\begin{array}{l}\text { Sensibilité } \\
\text { de l'isolat } \\
\text { responsable } \\
\text { d'infection }\end{array}$ & $\begin{array}{l}\text { Répond } \\
\text { partiellement } \\
\text { aux besoins }\end{array}$ & Faible \\
\hline
\end{tabular}

Abréviations : ERV, entérocoques résistants à la vancomycine; SARM, Staphylococcus aureus résistant à la méthicilline

des isolats responsables d'infection à $S$. aureus; sensibilité des isolats responsables d'infection à Neisseria gonorrhoeae); et une se trouvait dans les deux types de milieux (sensibilité des isolats responsables d'infection à Streptococcus pneumoniae [maladie invasive]). Dans l'évaluation de ce qui était nécessaire pour satisfaire aux exigences, six pouvaient être satisfaites à I'aide d'une faisabilité modérée ou élevée et une à l'aide d'une faisabilité faible. La mesure des données hautement prioritaires à faisabilité faible a été déterminée pour la sensibilité des isolats responsables d'infection à $N$. gonorrhoeae au sein de la collectivité.

\section{Ne répond pas aux besoins}

Le groupe de travail a ensuite examiné les deux des 19 mesures hautement prioritaires pour lesquelles les systèmes de surveillance existants à l'échelle nationale ne répondaient pas aux besoins (tableau 4). Ces deux mesures étaient liées aux taux d'infection à $C$. difficile dans le cadre de la surveillance communautaire et à la sensibilité des isolats responsables d'infection aux espèces Enterobacteriaceae. Dans les deux cas, la faisabilité afin des interventions requises pour combler les lacunes des données a été considérée comme étant modérée. 
Tableau 4 : Mesures de données hautement prioritaires pour lesquelles les systèmes de surveillance actuels ne répondent pas aux besoins

\begin{tabular}{|l|l|l|l|l|}
\hline \multicolumn{1}{|c|}{ Organisme } & Milieu & $\begin{array}{l}\text { Mesure de } \\
\text { donnée } \\
\text { prioritaire }\end{array}$ & $\begin{array}{l}\text { Système de } \\
\text { surveillance } \\
\text { actuel }\end{array}$ & Faisabilité \\
\hline $\begin{array}{l}\text { Clostridium difficile } \\
\text { (maladie } \\
\text { diarrhéique) }\end{array}$ & Communautaire & $\begin{array}{l}\text { Taux } \\
\text { d'infection }\end{array}$ & $\begin{array}{l}\text { Ne répond } \\
\text { pas aux } \\
\text { besoins }\end{array}$ & Modérée \\
\hline $\begin{array}{l}\text { Espèces } \\
\text { Enterobacteriaceae } \\
\begin{array}{l}\text { Escherichia coli } \\
\text { et Klebsiella } \\
\text { (infections } \\
\text { génito-urinaires) }\end{array}\end{array}$ & Communautaire & $\begin{array}{l}\text { Sensibilité } \\
\text { de l'isolat } \\
\text { responsable } \\
\text { d'infection }\end{array}$ & $\begin{array}{l}\text { Ne répond } \\
\text { pas aux } \\
\text { besoins }\end{array}$ & Modérée \\
\hline
\end{tabular}

\section{Exigences en matière de données modérément prioritaires}

Parmi les organismes de priorité de premier ordre, le groupe de travail a déterminé huit mesures de données de priorité modérée; quatre dans un milieu de soins de santé, trois dans des milieux communautaires et une dans des milieux de soins de santé ainsi que communautaires (tableau 5). Parmi celles-ci, on comptait une mesure de données pour laquelle les systèmes de surveillance existants répondaient entièrement aux besoins et cinq mesures de données (quatre dans des milieux hospitaliers), pour lesquelles les systèmes de surveillance actuels répondaient

\section{Tableau 5 : Mesures de données de priorité modérée}

\begin{tabular}{|c|c|c|c|c|}
\hline Organisme & Milieu & $\begin{array}{l}\text { Mesure de } \\
\text { donnée } \\
\text { prioritaire }\end{array}$ & $\begin{array}{c}\text { Système de } \\
\text { surveillance } \\
\text { actuel }\end{array}$ & Faisabilité \\
\hline $\begin{array}{l}\text { Clostridium difficile } \\
\text { (maladie diarrhéique) }\end{array}$ & Soins de santé & $\begin{array}{l}\text { Sensibilité } \\
\text { de l'isolat } \\
\text { responsable } \\
\text { d'infection }\end{array}$ & $\begin{array}{l}\text { Répond aux } \\
\text { besoins }\end{array}$ & Élevée \\
\hline $\begin{array}{l}\text { Espèces } \\
\text { Enterobacteriaceae } \\
\text { Escherichiae coli et } \\
\text { Klebsiella (sites de } \\
\text { colonisation) }\end{array}$ & Soins de santé & $\begin{array}{l}\text { Sensibilité } \\
\text { de l'isolat } \\
\text { responsable } \\
\text { de colonisation }\end{array}$ & $\begin{array}{l}\text { Répond } \\
\text { partiellement } \\
\text { aux besoins }\end{array}$ & Modérée \\
\hline $\begin{array}{l}\text { Espèces } \\
\text { Pseudomonas et } \\
\text { espèces Acinetobacter } \\
\text { (Infections du sang) }\end{array}$ & Soins de santé & $\begin{array}{l}\text { Sensibilité } \\
\text { de l'isolat } \\
\text { responsable } \\
\text { d'infection }\end{array}$ & $\begin{array}{l}\text { Répond } \\
\text { partiellement } \\
\text { aux besoins }\end{array}$ & Modérée \\
\hline $\begin{array}{l}\text { Espèces Pseudomonas } \\
\text { et espèces } \\
\text { Acinetobacter (sites } \\
\text { de colonisation) }\end{array}$ & Soins de santé & $\begin{array}{l}\text { Sensibilité } \\
\text { de l'isolat } \\
\text { responsable } \\
\text { de colonisation }\end{array}$ & $\begin{array}{l}\text { Répond } \\
\text { partiellement } \\
\text { aux besoins }\end{array}$ & Modérée \\
\hline $\begin{array}{l}\text { Espèces } \\
\text { Campylobacter }\end{array}$ & $\begin{array}{l}\text { Soins de } \\
\text { santé et } \\
\text { communautaire }\end{array}$ & $\begin{array}{l}\text { Sensibilité } \\
\text { de l'isolat } \\
\text { responsable } \\
\text { d'infection }\end{array}$ & $\begin{array}{l}\text { Répond } \\
\text { partiellement } \\
\text { aux besoins }\end{array}$ & Faible \\
\hline $\begin{array}{l}\text { Staphylococcus aureus } \\
\text { (autres sites } \\
\text { d'infection [y compris } \\
\text { les infections du } \\
\text { sang] et sites de } \\
\text { colonisation) }\end{array}$ & Communautaire & $\begin{array}{l}\text { Taux } \\
\text { d'infection }\end{array}$ & $\begin{array}{l}\text { Répond } \\
\text { partiellement } \\
\text { aux besoins }\end{array}$ & Faible \\
\hline $\begin{array}{l}\text { Clostridium difficile } \\
\text { (maladie diarrhéique) }\end{array}$ & Communautaire & $\begin{array}{l}\text { Sensibilité } \\
\text { de l'isolat } \\
\text { responsable } \\
\text { d'infection }\end{array}$ & $\begin{array}{l}\text { Ne répond } \\
\text { pas aux } \\
\text { besoins }\end{array}$ & Faible \\
\hline $\begin{array}{l}\text { Espèces } \\
\text { Enterobacteriaceae } \\
\text { E. coli et Klebsiella } \\
\text { (infections } \\
\text { génito-urinaires) }\end{array}$ & Communautaire & $\begin{array}{l}\text { Taux } \\
\text { d'infection }\end{array}$ & $\begin{array}{l}\text { Ne répond } \\
\text { pas aux } \\
\text { besoins }\end{array}$ & Faible \\
\hline
\end{tabular}

partiellement aux besoins. La seule mesure de données en milieu hospitalier qui répondait partiellement aux besoins était les taux d'infection à $S$. aureus et la faisabilité pour répondre à ce besoin en matière de données a été jugée comme étant faible. On a observé deux mesures de données, toutes deux dans des milieux communautaires, pour lesquelles le système de surveillance actuel ne répondait pas aux besoins. Il s'agissait de la sensibilité de l'isolat responsable d'infection à $C$. difficile et les taux d'infection pour les espèces Enterobacteriaceae, plus précisément, les infections génito-urinaires à E. coli et à Klebsiella.

\section{Exigences en matière de données faiblement prioritaires}

Parmi les organismes de priorité de premier ordre, le groupe de travail a mis en évidence 14 mesures de données faiblement prioritaires (données non indiquées). Actuellement, certains systèmes de surveillance recueillent entièrement ou partiellement ces mesures de données faiblement prioritaires et il a été reconnu que leur priorité continue pourrait nécessiter un examen.

\section{Recommandations}

Le groupe de travail a recommandé d'axer les efforts sur les mesures de données de priorité élevée et modérée lorsque les systèmes de surveillance actuels satisfont partiellement ou ne satisfont pas complètement aux exigences en matière de données et lorsque la faisabilité des interventions requises pour combler les lacunes décelées en matière de données est considérée comme étant modérée à élevée.

Les membres du groupe de travail sont parvenus au consensus que, compte tenu de leur taux de morbidité et de mortalité élevés dans les milieux de soins de santé, la principale priorité devrait être accordée à la surveillance de la résistance aux antimicrobiens des infections du sang aux espèces Enterococcus, aux espèces Enterobacteriaceae ( $E$. coli et Klebsiella) et à Staphylococcus aureus. Cette recommandation est conforme aux recommandations de l'OMS relatives à la surveillance de la résistance aux antimicrobiens (9). Bien que le système de surveillance national actuel recueille partiellement des données sur les organismes présentant un profil de résistance précis qui sont jugés actuellement comme les plus importants (p. ex. Staphylococcus aureus résistant à la méthicilline ou SARM), il a été remarqué qu'idéalement, le taux de toutes les infections du sang causées par ces organismes dans des milieux de soins de santé devraient être recueilli et, afin d'effectuer le suivi de la résistance émergente, tous les profils de sensibilité existants devraient également être indentifiés.

Pour les deux mesures de données hautement prioritaires dans les milieux communautaires, le groupe de travail a recommandé la réalisation d'une étude de prévalence ponctuelle au sein de la collectivité (c.-à-d. tous les cinq ans) pour effectuer le suivi les taux d'infection à $C$. difficile ainsi que des données d'antibiogrammes à l'échelle des collectivités annuellement pour les données de sensibilité pour les espèces Enterobacteriaceae (E. coli et Klebsiella) causant des infections génito-urinaires. En outre, il a été recommandé qu'on accorde une priorité élevée 
à la collecte de données sur la sensibilité communautaire pour $N$. gonorrhoeae compte tenu de l'évolution de sa résistance et du fait que le système de surveillance actuel ne recueille que partiellement ces données. Cependant, la faisabilité de la collecte plus entière de ces données a été jugée faible compte tenu des limites logistiques et cliniques.

Dans l'ensemble, le groupe de travail a aussi recommandé de procéder à un examen des priorités relatives aux exigences en matière de données de surveillance de la résistance aux antimicrobiens à l'échelle nationale à mesure que de nouveaux problèmes émergent et de façon régulière. D'autres recommandations relatives aux exigences en matière de données de priorité modérée et faible se trouvent dans le rapport complet.

\section{Discussion}

Le groupe de travail chargé de la surveillance de la résistance aux antimicrobiens du CDMTI a mené une évaluation approfondie et a utilisé un processus de priorisation afin de déterminer les exigences en matière de données de surveillance de la résistance aux antimicrobiens prioritaires à l'échelle nationale ainsi que les lacunes en matière de données sur les organismes résistants aux antimicrobiens de priorité de premier ordre. Il a constaté que, pour un peu plus de la moitié des mesures de données hautement prioritaires déterminées (10/19), les systèmes de surveillance existants répondaient aux besoins.

Lors de la comparaison des données de surveillance pour les organismes résistants aux antimicrobiens de priorité de premier ordre dans différents milieux, les données sur la résistance aux antimicrobiens à l'échelle communautaire ont été déterminées comme présentant des lacunes importantes aux fins de la surveillance nationale. Actuellement, ce manque de données de surveillance entraîne des lacunes en matière de connaissances dans la compréhension du fardeau des infections résistantes aux antimicrobiens au sein des milieux communautaires. Les infections du sang ont été désignées comme le site d'infection possédant la priorité principale en matière de surveillance de la résistance aux antimicrobiens pour plusieurs organismes dans les milieux de soins de santé. Étant donné que la résistance et le recours aux antimicrobiens évoluent constamment, le groupe de travail a recommandé de procéder à un examen des priorités relatives aux exigences en matière de données de surveillance de la résistance aux antimicrobiens à l'échelle nationale de façon régulière et lorsque les nouveaux problèmes émergent.

Le rapport du groupe de travail a été soumis au Conseil du Réseau pancanadien de santé publique et fait actuellement l'objet d'un examen. Par l'entremise du mandat du Conseil du Réseau pancanadien de santé publique, les prochaines étapes comprendront l'établissement des rôles et des responsabilités des gouvernements fédéral, provinciaux et territoriaux pour combler ces lacunes.

\section{Remerciements}

Les auteurs tiennent à remercier sincèrement le travail de tous les membres du groupe de travail chargé de la résistance aux antimicrobiens de 2015 du CDMTI :

John Conly, Université de Calgary; Charles Frenette, Université McGill; Greg German, ministère de la Santé de l'île-du-Prince-Édouard; Jennie Johnstone, Santé publique Ontario; Paul N. Levett, Saskatchewan Disease Control Laboratory; Warren Mclsaac, Université de Toronto (jusqu'en mars 2015); Jessica Minion, Regina General Hospital, Lindsay E. Nicolle, Université du Manitoba; David Patrick, Centre de contrôle des maladies de la Colombie-Britannique; Virginia Roth, Université d'Ottawa (jusqu'en avril 2015); Nadine Sicard, ministère de la Santé et des Services sociaux du Québec; Kimberley Simmonds, ministère de la Santé de l'Alberta.

Agence de la santé publique du Canada : Kanchana Amaratunga, Chris Archibald (président), Jacqueline Arthur (Secrétariat), Christine Cryan (Secrétariat), Rita Finley, Rebecca Irwin, Michael Mulvey, Jill Tarasuk (Secrétariat).

\section{Conflit d'intérêts}

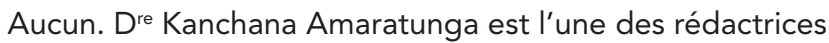
invitées pour la présente publication et se récuse des décisions de la rédaction relatives au présent article. Les décisions de la rédaction ont été prises par la rédactrice en chef, Dre Patricia Huston, et l'autre rédactrice invitée, Jacqueline Arthur.

\section{Financement}

Ce travail a été appuyé par l'Agence de la santé publique du Canada.

\section{Références}

1. Gravel D, Archibald CP, Pelude L, Mulvey M, Golding G. Surveillance de la résistance aux antimicrobiens dans les hôpitaux canadiens, 2007-2012. Relevé des maladies transmissibles au Canada 2014;40(S2):6-13. Disponible à l'adresse : http://www.phac-aspc.gc.ca/publicat/ccdrrmtc/14vol40/dr-rm40s-2/dr-rm40s-2-surv-1-fra.php.

2. Finley R. Recommandations d'antibiotiques par les médecins de cabinet médicaux, 2007-2011. Relevé des maladies transmissibles au Canada. 2014;40(S2):14-22. Disponible à l'adresse : http://www.phac-aspc.gc.ca/publicat/ccdrrmtc/14vol40/dr-rm40s-2/dr-rm40s-2-surv-2-fra.php.

3. Finley R. Achat d'antibiotiques par les hôpitaux canadiens, 2007-2011. Relevé des maladies transmissibles au Canada. 2014;40(S2):23-28. Disponible à l'adresse : http://www.phacaspc.gc.ca/publicat/ccdr-rmtc/14vol40/dr-rm40s-2/dr-rm40s2-surv-3-fra.php. 
4. Avery BP, Parmley EJ, Reid-Smith RJ, Daignault D, Finley $\mathrm{RL}$, Irwin RJ. Programme intégré canadien de surveillance de la résistance aux antimicrobiens : Faits saillants aliments vendus au détail, 2003-2012. Relevé des maladies transmissibles au Canada. 2014;40(S2):29-35. Disponible à l'adresse : http://www.phac-aspc.gc.ca/publicat/ccdrrmtc/14vol40/dr-rm40s-2/dr-rm40s-2-surv-4-fra.php.

5. Agence de la santé publique du Canada en collaboration avec l'Agence canadienne d'inspection des aliments, les Instituts de recherche en santé du Canada, Santé Canada, Agriculture et Agroalimentaire Canada, Industrie Canada et le Conseil national de recherches Canada. Plan d'action fédéral sur la résistance et le recours aux antimicrobiens au Canada. Relevé des maladies transmissibles au Canada. 2015;41(S4):19-22. Disponible à l'adresse : http://www.phacaspc.gc.ca/publicat/ccdr-rmtc/15vol41/dr-rm41s-4/overviewapercu_04-fra.php.

6. Ebrahim M, Gravel D, Thabet C, Abdesselam K, Paramalingam S, Hyson C. Tendances liées à I'utilisation des antimicrobiens et à la résistance aux antimicrobiens au Canada : 2014. Relevé des maladies transmissibles au Canada. 2016;42(11):251-6. Disponible à l'adresse : http:// www.phac-aspc.gc.ca/publicat/ccdr-rmtc/16vol42/drrm42-11/ar-02-fra.php.
7. Réseau pancanadian de santé publique, Groupe de travail chargé de la surveillance de la résistance aux antimicrobiens de Comité directeur sur les maladies transmissibles et infectieuses. [Internet]. Rapport au CDMTI : Données de surveillance exigées concernant la résistance aux antimicrobiens des organismes prioritaires. Ottawa ON: RSPC; 2016 avr. Disponible à l'adresse : https://www. phn-rsp.ca/fr/rapports-publications/donnees-surveillanceexigees-resistance-antimicrobiens-organismes-prioritaires. html.

8. World Health Organization (WHO) [Internet]. Global Antimicrobial Resistance Surveillance System: Manual for early implementation. Geneva: WHO; 2015. Disponible à l'adresse : http://apps.who.int/iris/ bitstream/10665/188783/1/9789241549400_eng.pdf.

9. World Health Organization (WHO) [Internet]. Antimicrobial resistance: Global report on surveillance. Geneva: WHO; 2014. Available from: http://www.who.int/drugresistance/ documents/surveillancereport/en/.

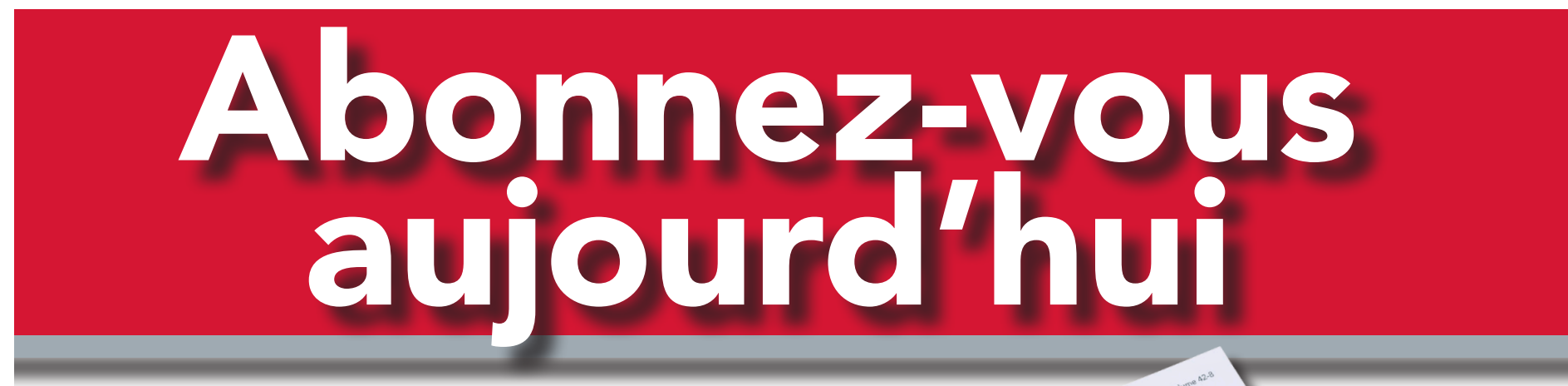

\section{Abonnez-vous au RMTC en ligne et recevez les nouveaux numéros directement dans votre boîte de réception.}

Recherche web

RMTC-abonnez-vous
Q

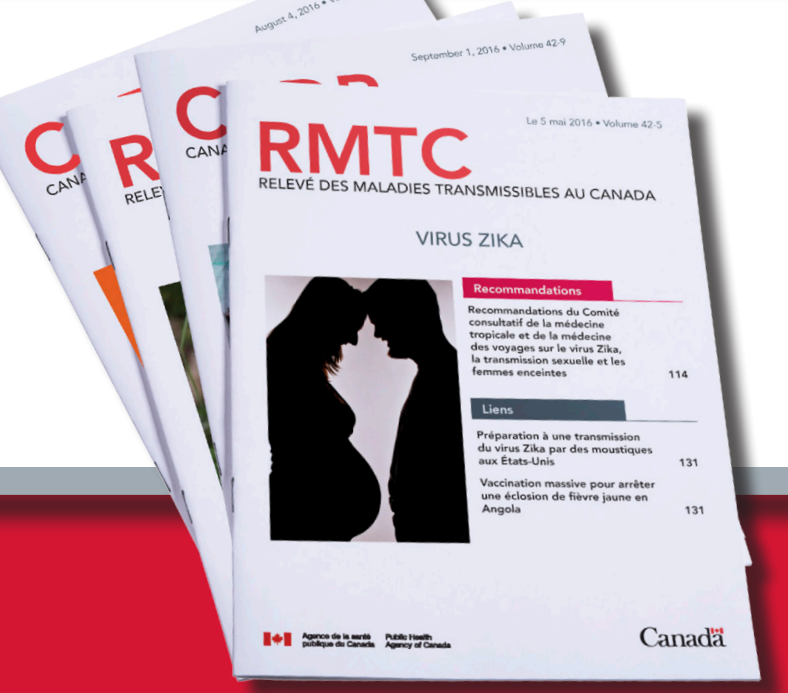

